\title{
Tender, Diffuse, Edematous, and Erythematous Papules on the Face, Neck, Chest, and Extremities
}

\author{
Christine Schleich, MD; Tammie Ferringer, MD
}

Eligible for 1 MOC SA Credit From the ABD

This Dermatopathology Diagnosis in our print edition is eligible for 1 self-assessment credit for Maintenance of Certification from the American Board of Dermatology (ABD). After completing this activity, diplomates can visit the ABD website (http://www.abderm.org) to self-report the credits under the activity title "Cutis Dermatopathology Diagnosis." You may report the credit after each activity is completed or after accumulating multiple credits.

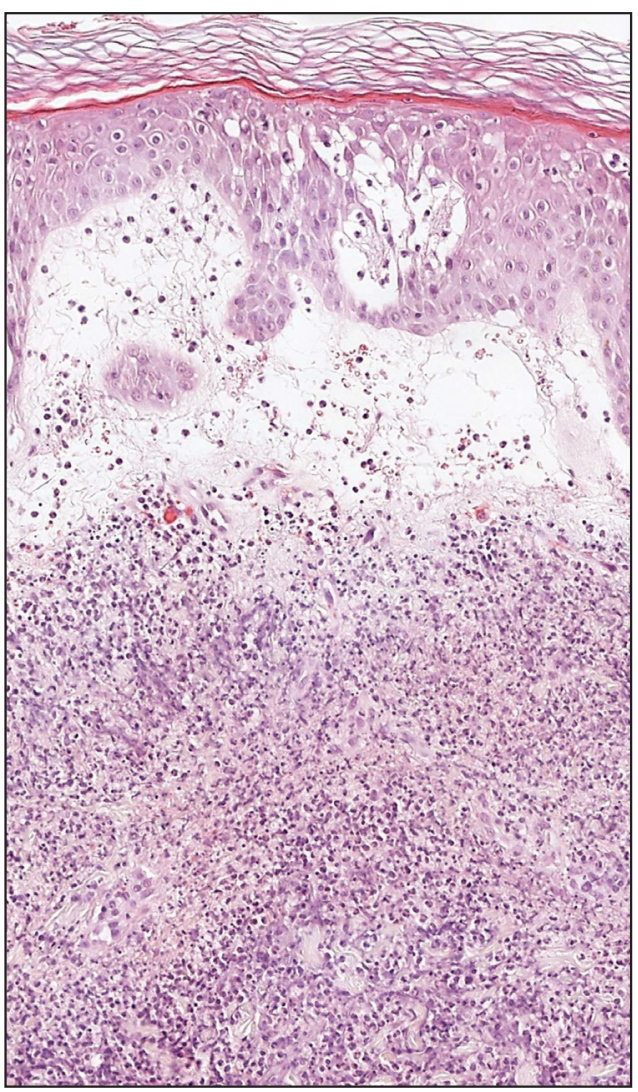

$\mathrm{H} \& \mathrm{E}$, original magnification $\times 20$
A 62-year-old woman presented with a tender diffuse eruption of erythematous and edematous papules and plaques on the face, neck, chest, and extremities, some appearing vesiculopustular.

\section{THE BEST DIAGNOSIS IS:}

a. bullous tinea corporis

b. perniosis

c. polymorphous light eruption

d. Sweet syndrome

e. Wells syndrome

PLEASE TURN TO PAGE 197 FOR THE DIAGNOSIS

From the Departments of Dermatology and Laboratory Medicine, Geisinger Medical Center, Danville, Pennsylvania.

The authors report no conflict of interest.

Correspondence: Christine Schleich, MD, Department of Dermatology, Geisinger Medical Center, 16 Woodbine Ln, Danville, PA 17821 (caschleich@geisinger.edu).

doi:10.12788/cutis.0218 


\section{THE DIAGNOSIS: Sweet Syndrome}

S weet syndrome, alternatively known as acute febrile neutrophilic dermatosis, typically presents with variably tender, erythematous papules, plaques, or nodules in middle-aged adults. ${ }^{1}$ Systemic symptoms such as fever, fatigue, and arthralgia often accompany these cutaneous findings. ${ }^{1,2}$ Although the pathophysiology has not been fully elucidated, this syndrome frequently is associated with infections, especially upper respiratory illnesses; medications; and malignancies. Among cases of malignancy-associated Sweet syndrome, hematologic malignancies, particularly acute myeloid leukemia and myelodysplastic syndrome, are more common than solid organ malignancies. ${ }^{1,2}$ Sweet syndrome may precede the associated malignancy by several months; thus, patients without an identifiable trigger for Sweet syndrome should be closely followed. ${ }^{2}$ Treatment with systemic steroids typically is effective. ${ }^{1,3}$ Typical histologic features include papillary dermal edema and a brisk neutrophilic infiltrate in the superficial to mid dermis (quiz image). ${ }^{4}$ Overlying epidermal spongiosis with or without vesiculation also can be seen. ${ }^{4}$ Leukocytoclasia and endothelial swelling without fibrinoid necrosis are typical, though full-blown leukocytoclastic vasculitis can be seen. ${ }^{3,4}$ A histiocytoid variant also has been described in which the dermal infiltrate is composed of mononuclear cells reminiscent of histiocytes that are thought to be immature cells of myeloid origin. This variant histologically can simulate leukemia cutis. ${ }^{5}$

Perniosis, also known as chilblains, typically presents with red to violaceous macules or papules on acral sites, particularly the distal fingers and toes. ${ }^{6,7}$ It tends to affect young women more frequently than other demographic groups. Although the pathophysiology is not fully understood, perniosis is thought to represent an abnormal inflammatory response to cold environmental conditions. It can occur as an idiopathic disorder or in association with various systemic illnesses including lupus erythematosus. ${ }^{6,7}$ The typical histologic findings include papillary dermal edema and a lymphocytic infiltrate in the superficial to deep dermis, often with perivascular and perieccrine accentuation (Figure 1). ${ }^{3,6}$ Other less common microscopic findings include sparse keratinocyte necrosis, basal layer vacuolar change, swelling of endothelial cells, and lymphocytic vasculitis. ${ }^{6}$ The lesions typically resolve spontaneously within a few weeks, but in some cases they may be chronic. ${ }^{3}$

Polymorphous light eruption, a common photodermatosis induced by UV light exposure, typically presents in adolescence or early adulthood with a female predominance. Patients usually develop this pruritic rash on sunexposed skin other than the face and dorsal aspects of the hands in the spring or early summer upon increased sun exposure after the winter season. ${ }^{3,8}$ Consistent sunlight exposure throughout the summer months results in decreased flares. Various cutaneous morphologies including papules, vesicles, and plaques can be seen., Histologic findings include papillary dermal edema and a perivascular lymphocytic infiltrate in the superficial to deep dermis (Figure 2). ${ }^{4}$

Tinea corporis, a superficial cutaneous dermatophyte infection, typically presents, as annular scaly plaques with central clearing. Vesicles and pustules also can be seen. ${ }^{3}$ The diagnosis can be confirmed via fungal culture, identification of hyphae on microscopic examination of skin scrapings using potassium hydroxide, or cutaneous biopsy. Histologic clues to diagnosis include a "compact stratum corneum (either uniform or forming a layer beneath a basket weave stratum corneum), parakeratosis, mild spongiosis, and neutrophils in the stratum corneum" (Figure 3). 9 Papillary dermal edema also may be present, though this finding less commonly is reported..$^{9,10}$ Because fungal hyphae can be difficult to identify on hematoxylin

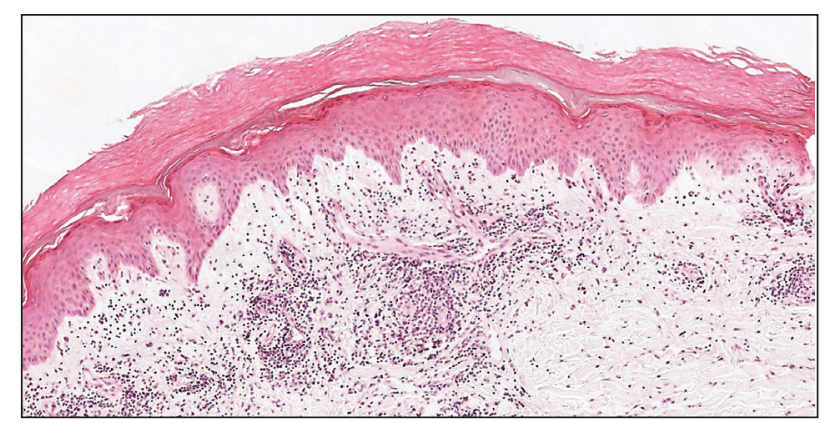

FIGURE 1. Perniosis. Hyperkeratosis consistent with acral skin, papillary dermal edema, and a perivascular lymphocytic infiltrate (H\&E, original magnification $\times 10$ ).

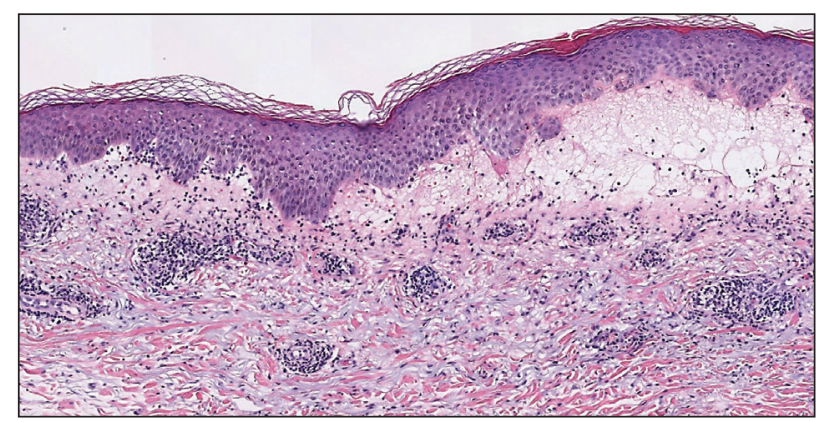

FIGURE 2. Polymorphous light eruption. Papillary dermal edema and a perivascular lymphocytic infiltrate $(H \& E$, original magnification $\times 10)$. 


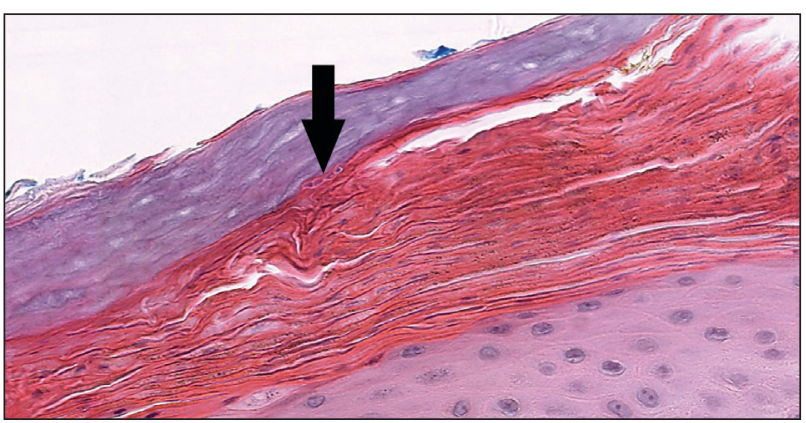

FIGURE 3. Tinea corporis. Fungal hyphae (arrow) present at the interface of compact orthokeratosis and parakeratosis (H\&E, original magnification $\times 40$ ).

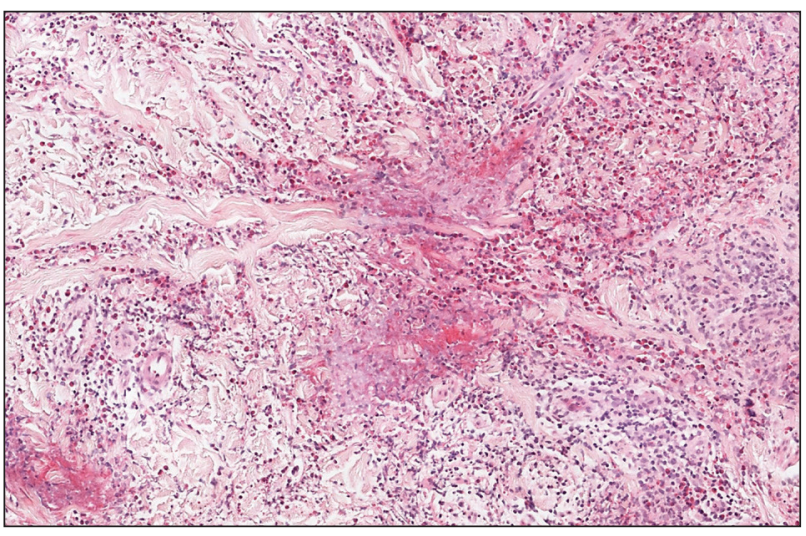

FIGURE 4. Wells syndrome. A mixed inflammatory infiltrate with many eosinophils and flame figures (H\&E, original magnification $\times 20)$.

and eosin-stained slides, special stains such as periodic acid-Schiff or Grocott-Gomori methenamine-silver may be helpful. ${ }^{9}$ These infections are managed with topical or oral antifungal medications.

Wells syndrome, also known as eosinophilic cellulitis, presents with an acute eruption that can clinically resemble bacterial cellulitis. ${ }^{3}$ It has been described in children and adults with various clinical morphologies including plaques, bullae, papulovesicles, and papulonodules. Peripheral eosinophilia may be present. ${ }^{11}$ The clinical lesions usually resolve spontaneously in a few weeks to months, but recurrences are typical. ${ }^{3,11}$ Histologic findings include papillary dermal edema with or without subepidermal bulla formation and epidermal spongiosis as well as a mixed inflammatory infiltrate with a predominance of eosinophils and flame figures (Figure 4). ${ }^{4}$ Flame figures are collagen fibers coated with major basic protein and other constituents of degranulated eosinophils. ${ }^{3}$ Although flame figures often are present in Wells syndrome, they are not specific to this condition. ${ }^{3,4}$ Some consider Wells syndrome an exaggerated reaction pattern rather than a specific entity. ${ }^{3}$

\section{REFERENCES}

1. Rochet N, Chavan R, Cappel M, et al. Sweet syndrome: clinical presentation, associations, and response to treatment in 77 patients. J Am Acad Dermatol. 2013;69:557-564.

2. Marcoval J, Martín-Callizo C, Valentí-Medina F, et al. Sweet syndrome: long-term follow-up of 138 patients. Clin Exp Dermatol. 2016;41:741-746.

3. Bolognia JL, Jorizzo JL, Shaffer JV. Dermatology. 3rd ed. Elsevier; 2012.

4. Calonje JE, Brenn T, Lazar AJ, et al. McKee's Pathology of the Skin. 4th ed. Elsevier Saunders; 2012.

5. Alegría-Landa V, Rodríguez-Pinilla S, Santos-Briz A, et al. Clinicopathologic, immunohistochemical, and molecular features of histiocytoid Sweet syndrome. JAMA Dermatol. 2017;153:651-659.

6. Boada A, Bielsa I, Fernández-Figueras M, et al. Perniosis: clinical and histopathological analysis. Am J Dermatopathol. 2010;32:19-23.

7. Takci Z, Vahaboglu G, Eksioglu H. Epidemiological patterns of perniosis, and its association with systemic disorder. Clin Exp Dermatol. 2012;37:844-849.

8. Gruber-Wackernagel A, Byrne S, Wolf P. Polymorphous light eruption: clinic aspects and pathogenesis. Dermatol Clin. 2014;32:315-334.

9. Elbendary A, Valdebran M, Gad A, et al. When to suspect tinea; a histopathologic study of 103 cases of PAS-positive tinea. J Cutan Pathol. 2016;46:852-857.

10. Hoss D, Berke A, Kerr P, et al. Prominent papillary dermal edema in dermatophytosis (tinea corporis). J Cutan Pathol. 2010;37:237-242.

11. Caputo R, Marzano A, Vezzoli P, et al. Wells syndrome in adults and children: a report of 19 cases. Arch Dermatol. 2006;142:1157-1161. 